\title{
Radiofrequency Catheter Ablation of AV Nodal Reentrant Tachycardia in Situs Inversus Totalis
}

SREEDHAR BILLAKANTY, M.D., THOMAS CRAWFORD, M.D., ERIC GOOD, M.D., and HAKAN ORAL, M.D.

From the Division of Cardiovascular Medicine, University of Michigan, Ann Arbor, Michigan

A 41-year-old male with situs inversus totalis had paroxysmal supraventricular tachycardia. On electrophysiology study, the mechanism of the tachycardia was determined as slow/fast atrioventricular nodal reentrant tachycardia and slow pathway was successfully performed under fluoroscopic guidance. (PACE 2009; 32:403-405)

\section{catheter ablation, dextrocardia, situs inversus, supraventricular tachycardia}

\section{Case Report}

A 41-year-old man with situs inversus totalis presented with a long-standing history of palpitations. A narrow QRS complex tachycardia at a ventricular rate of $200 \mathrm{bpm}$ was captured on an event monitor. A standard 12-lead electrocardiogram (ECG) in sinus rhythm is shown in Figure 1.

After having informed consent, an electrophysiological study was performed in the postabsorptive state under conscious sedation. Vascular access was obtained through a right femoral vein. Projections mirroring conventional fluoro-

Address for reprints: Hakan Oral, M.D., Cardiovascular Center, SPC 5853, 1500 East Medical Center Drive, Ann Arbor, MI 48109-5853. Fax: 734-936-7026; e-mail: oralh@umich.edu

Received April 22, 2008; revised May 19, 2008; accepted June 10, 2008. scopic views were used to guide catheter navigation. Cardiac chambers were identified by electrogram and fluoroscopic guidance (Fig. 2). Two quadripolar electrode catheters were positioned in the high right atrium and right ventricular apex, respectively. A temperature-controlled 4mm-tip steerable catheter was advanced to the Hisbundle position. Programmed stimulation of the atrium demonstrated dual atrioventricular (AV) nodal physiology, and a narrow QRS complex tachycardia at a cycle length of $260 \mathrm{~ms}$ was readily inducible (Fig. 3). A short septal VA time of $35 \mathrm{~ms}$ and a $\mathrm{V}-\mathrm{A}-\mathrm{V}$ response to entrainment of the tachycardia from the ventricle confirmed the mechanism as slow/fast AV nodal reentrant tachycardia. Slow pathway ablation was performed at the roof of the coronary sinus ostium where the local $\mathrm{A} / \mathrm{V}$ was $<0.5$, and resulted in junctional ectopy during application of radiofrequency energy. After the ablation, there was no residual slow

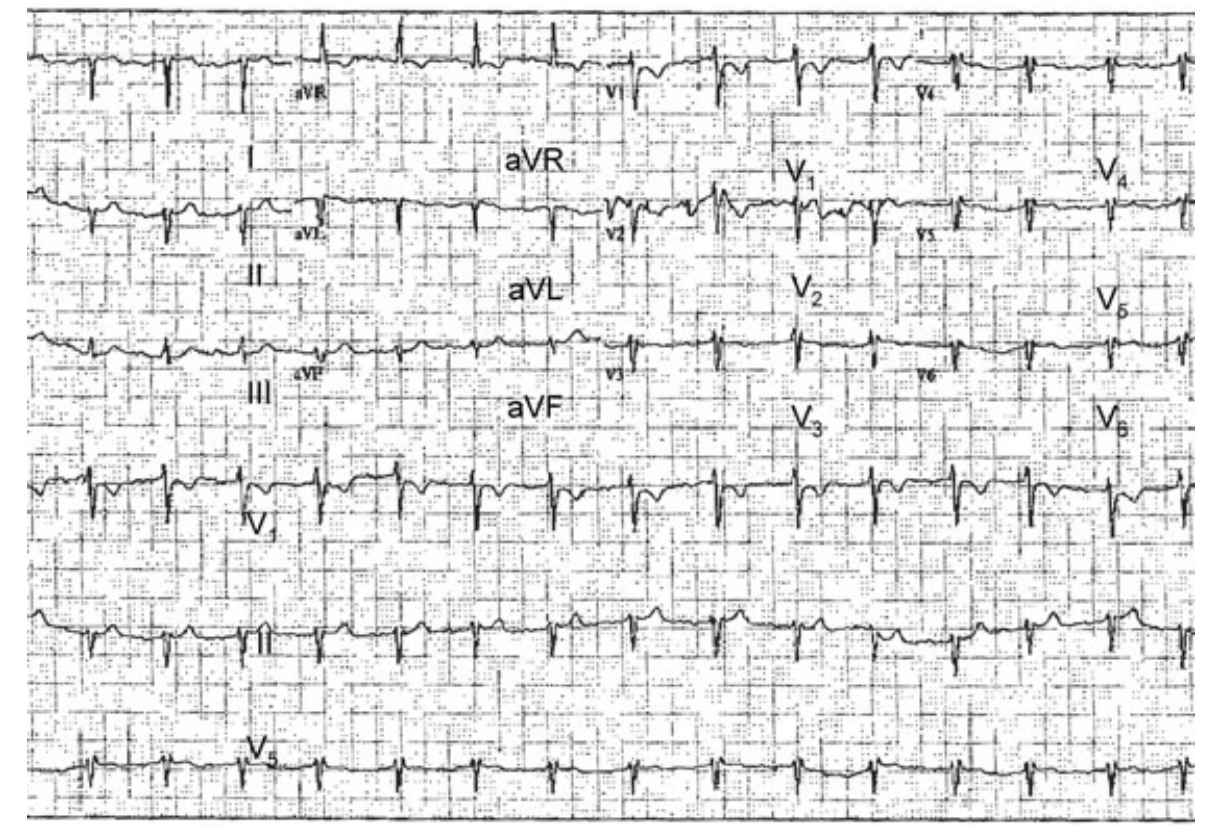

Figure 1. Standard 12-lead ECG. Right-ward axis of the P wave and QRS complexes in the frontal plane and poor $R$-wave progression in precordial leads are noted.

(C)2009, The Authors. Journal compilation (C)2009 Wiley Periodicals, Inc. 
A

B
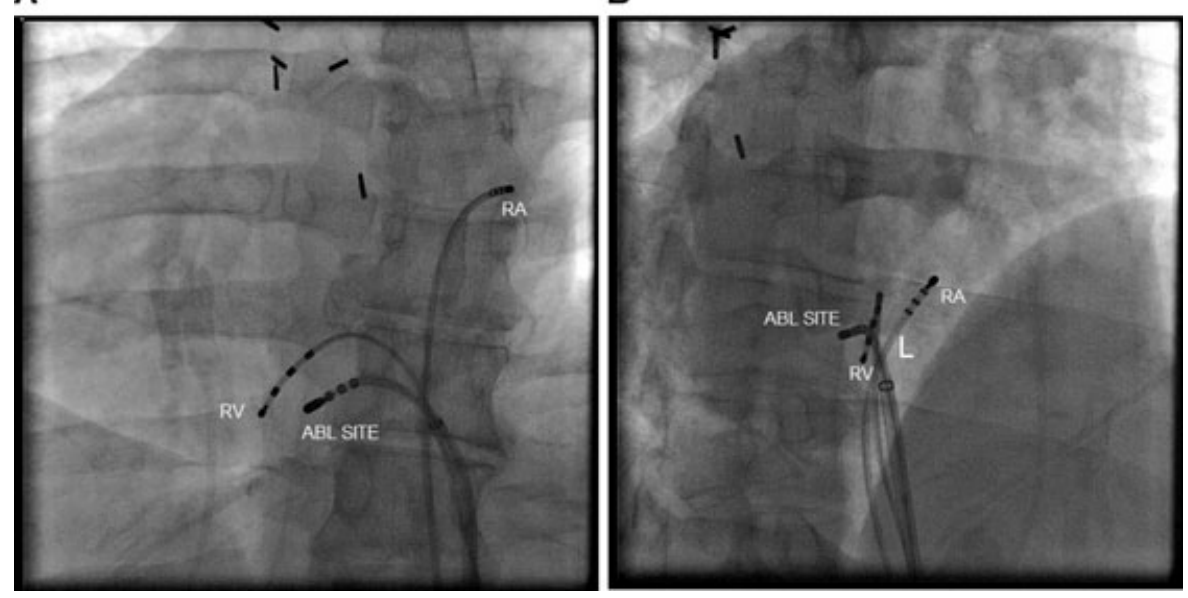

Figure 2. Fluoroscopic projections. Shown are catheter positions in left anterior oblique (LAO) 30-degree (Panel A) and right anterior oblique (RAO) 50-degree (Panel B) views to mirror standard RAO 30-degree and LAO 50-degree projections, respectively. Abbreviations: HRA $=$ high right atrium, $H I S=$ his-bundle, $R V A=$ right ventricular apex, $L=$ left of the patient, $R=$ right of the patient.

pathway conduction and no supraventicular tachycardia was inducible without and with infusion of isoproterenol. There were no complications. The fluoroscopy and procedure times were 15 and 90 minutes, respectively.
There has been limited experience with radiofrequency catheter ablation in patients with dextrocardia. Successful catheter ablation of atrial tachycardia, atrial flutter, and an accessory pathway has been reported in patients with

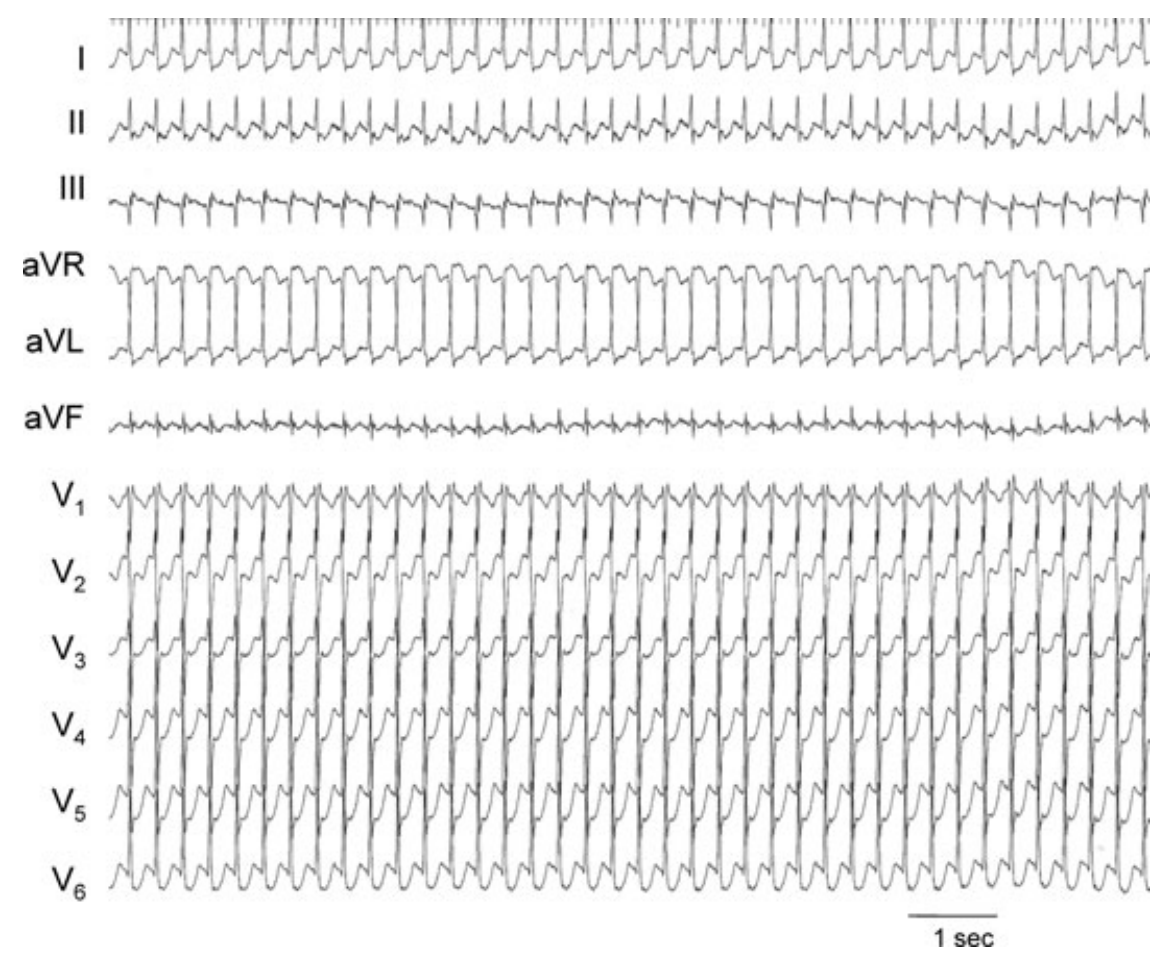

Figure 3. Twelve-lead ECG of the tachycardia with right-sided precordial leads. The limb leads were also reversed. The tachycardia was induced in the electrophysiology laboratory. 
dextrocardia. ${ }^{1-6}$ AV nodal reentrant tachycardia was also reported in a patient with dextrocardia who had single aortic coronary ostium, however, ablation was not attempted. ${ }^{7}$ In a prior study, slow pathway ablation in a patient with dextrocardia was associated with a long procedure time. ${ }^{8}$ In another study, successful slow pathway ablation was reported in a patient with dextrocardia, inferior vena cava interruption, and azygos continuation. ${ }^{9}$

\section{References}

1. Hatala R, Weiss C, Koschyk DH, Siebels J, Cappato R, Kuck KH. Radiofrequency catheter ablation of left atrial tachycardia originating within the pulmonary vein in a patient with dextrocardia. Pacing Clin Electrophysiol 1996; 19:999-1002.

2. Wu TJ, Chen SA, Chiang CE, Yang CJ, Cheng CC, Wang SP, Chiang BN, et al. Radiofrequency catheter ablation of sustained intraatrial reentrant tachycardia in a patient with mirrorimage dextrocardia. J Cardiovasc Electrophysiol 1994; 5:790794.

3. Abe H, Araki M, Nagatomo T, Miura Y, Nakashima Y. Radiofrequency catheter ablation of an accessory pathway in dextrocardia. Pacing Clin Electrophysiol 1997; 20(9 Pt 1):2284-2285.

4. Vaseghi M, Cesario D, Swerdlow CD, Shivkumar K. Counterclockwise atrial flutter in dextrocardia. Heart Rhythm 2005; 2:673-674.

5. Taniguchi H, Miyauchi Y, Kobayashi Y, Hirasawa Y, Hosaka H, Iwasaki YK, Katoh T, et al. Radiofrequency catheter ablation of
This study illustrates that typical features on a standard 12-lead ECG may hint to the presence of dextrocardia. Furthermore, electrophysiological study and successful catheter ablation can safely and efficiently be performed under fluoroscopy and electrogram guidance in patients with dextrocardia. Catheter ablation should be considered and offered as a therapeutic option to patients with supraventricular tachycardia and dextrocardia.

a coronary sinus-ventricular accessory connection in dextrocardia with complete situs inversus and an anomalous inferior vena cava. Pacing Clin Electrophysiol 2005; 28:164-167.

6. Huang FR, Hau DK, Lee MA. Radiofrequency catheter ablation of "typical" atrial flutter in a patient with Kartagener's syndrome. Pacing Clin Electrophysiol 2002; 25:1138-1141.

7. Yip AS, Chow WH, Cheung KL. Dextrocardia with single coronary artery ostium in a patient with aortic regurgitation and supraventricular tachycardia. A case history. Angiology 1994; 45:907-910.

8. Reithmann C, Hoffmann E, Dorwarth U, Remp T, Steinbeck G. Slow pathway ablation in a patient with common AV nodal reentrant tachycardia and complete situs inversus. Europace 1999; 1:283-285.

9. Pecoraro R, Proclemer A, Pivetta A, Gianfagna P. Radiofrequency ablation of atrioventricular nodal tachycardia in a patient with dextrocardia, inferior vena cava interruption, and azygos continuation. J Cardiovasc Electrophysiol 2008; 19:444. 